\title{
Space-time Averages in Macroscopic Gravity and Volume-preserving Coordinates
}

\author{
Marc Mars ${ }^{\natural *}$ and Roustam M. Zalaletdinov ${ }^{b \sharp \dagger \ddagger \S}$ \\ ${ }^{\natural}$ School of Mathematical Sciences, Queen Mary $\&$ Westfield College \\ University of London, Mile End Road, London E1 4NS, England, U.K. \\ ${ }^{b}$ N. Copernicus Astronomical Center, Polish Academy of Sciences \\ ul. Bartycka 18, 00-716 Warsaw, Poland \\ ${ }^{\sharp}$ Department of Theoretical Physics, Institute of Nuclear Physics \\ Uzbek Academy of Sciences, Tashkent 702132, Uzbekistan, C.I.S.
}

31 January 1997

\begin{abstract}
The definition of the covariant space-time averaging scheme for the objects (tensors, geometric objects, etc.) on differentiable metric manifolds with a volume $n$-form, which has been proposed for the formulation of macroscopic gravity, is analyzed. An overview of the space-time averaging procedure in Minkowski spacetime is given and comparison between this averaging scheme and that adopted in macroscopic gravity is carried out throughout the paper. Some new results concerning the algebraic structure of the averaging operator are precisely formulated and proved, the main one being that the averaging bilocal operator is idempotent iff it is factorized into a bilocal product of a matrix-valued function on the manifold, taken at a point, by its inverse at another point. The previously proved existence theorems for the averaging and coordination bilocal operators are revisited with more detailed proofs of related results. A number of new results concerning the structure of the volume-preserving averaging operators and the class of proper coordinate systems are given. It is shown, in particular, that such operators are defined on an arbitrary $n$-dimensional differentiable metric manifold with a volume $n$-form up to the freedom of $(n-1)$ arbitrary functions of $n$ arguments and 1 arbitrary function of $(n-1)$ arguments. All the results given in this paper are also valid whenever appropriate for affine connection manifolds including (pseudo)-Riemannian manifolds.
\end{abstract}

PACS Numbers: 04.20.Cv, 02.40.-k, 04.40.-b

*E-mail address: M.Mars@qmw.ac.uk

${ }^{\dagger}$ E-mail address: zala@camk.edu.pl

¥Śniadeccv Fellow 


\section{Introduction}

Space-time averaging procedures play an important role in modern physics because of, first of all, their relevance to deriving classical macroscopic theories. A well-known example of such a procedure is the space-time scheme developed for averaging out the microscopic Lorentz electrodynamics to derive the macroscopic Maxwell electrodynamics (see, for example, [1][4]. Another important physical argument for considering space-time averaging procedures is that they are relevant to modelling the process of physical measurement. It is the spacetime averages of physical fields that are known [10], [1] to have direct observational status and physical meaning.

The space-time averaging procedure of classical electrodynamics utilizes explicitly the flat character of the Minkowski space-time manifold and its formulation is essentially based on the existence of Cartesian coordinates. In this connection, the following questions are of interest and importance: whether it is possible $(a)$ to encode the properties of the averaging procedure in a covariant manner suitable for differentiable manifolds not equipped with a metric and a connection in general, and $(b)$ to formulate the corresponding covariant averaging procedure for the objects which can live on such manifolds and to clear up its geometric meaning. Having replied $(a)$ and $(b)$, the next question of primary importance is (c) how the averaging procedure may be made compatible with a metric and a connection when the differentiable manifold possesses those structures.

It should be pointed out here that the above problem of generalizing the flat space-time procedure for curved manifolds goes far beyond being simply an academic problem. One of its most important areas of applicability is the general theory of relativity where the space-time is a 4-dimensional pseudo-Riemannian manifold. As is well-known, there is not yet a satisfactory derivation of the Maxwell equations in general relativity, apart from the covariantization procedure where the partial derivatives of the special theory of relativity are replaced by covariant ones (see, for example, [12]). A physically motivated and mathematically correct derivation of the macroscopic Maxwell equations by averaging out the general relativistic microscopic Maxwell-Lorentz equations is still lacking and, furthermore, the very foundations of microscopic electrodynamics in general relativity are not well-established yet. Another problem in general relativity where the availability of a space-time averaging procedure is of primary importance is the so-called averaging problem (see [9], [13]- [15] for a review and discussion). Its main motivation comes from cosmology where Einstein's equations are usually utilized with a hydrodynamic stress-energy tensor without any satisfactory proof of why the left-hand side of the equations (the field operator) keeps the same structure while the right-hand side has been changed, or averaged, from a real discrete matter distribution (stars, galaxies, etc.) to a continuous one. The task here is to carry out a space-time averaging of Einstein's equations in order to understand the structure of the averaged (macroscopic) field equations and apply them to deal with the overwhelming majority of cosmological problems. A solution for this problem is also desirable in order to provide a rigorous basis for constructing continuous matter models from discrete ones (this construction is based in modern cosmology mainly on phenomenological grounds).

The goal of this paper is twofold: (1) to give precise formulations and detailed proofs of some new results concerning the algebraic structure of the averaging and coordination oper-

${ }^{1}$ Alternative approaches for deriving macroscopic electrodynamics which apply other averaging proce- 
ators and the properties of the space-time averages within the so-called macroscopic gravity [9], [14, [16]-19] (the basics of this averaging procedure has been developed in [20], [21]); (2) to revisit the previously shown existence of the bilocal operators of macroscopic gravity [14, [16] with more detailed proofs of related results. The paper is organized as follows. Section 2 gives an overview of the space-time averaging scheme used in electrodynamics. The space-time averages of macroscopic gravity are defined in Section 3. The next two sections are devoted to the algebra of the averaging operator with a discussion of its algebraic properties and a formulation of an important theorem stating that the averaging bilocal operator is idempotent iff it is factorized into a bilocal product of a matrix-valued function on the manifold, taken at a point, by its inverse at another point. The differential properties of the averages and the coordination bivector are summarized in Section 6. In Section 7 the existence theorems for the averaging and coordination operators are formulated and proved. The last Section 8 is devoted to the definition and properties of the proper coordinate systems, the coordinates in which the averaging operators take their simplest form. It is shown, in particular, that such operators are defined on an arbitrary $n$-dimensional differentiable metric manifold with a volume $n$-form up to the freedom of $(n-1)$ arbitrary functions of $n$ arguments and one arbitrary function of $(n-1)$ arguments. A summary on the meaning and content of the space-time averaging procedure of macroscopic gravity is given in the Conclusion.

Though given for $n$-dimensional differentiable metric manifolds with a volume $n$-form, they are valid whenever appropriate for affine connection manifolds, including (pseudo)-

Riemannian manifolds. All the results in this paper also hold generally for $n$-dimensional differentiable manifolds with a volume $n$-form possessing neither a metric nor a connection.

\section{Space-time averages in Minkowski manifold}

The space-time averaging procedure applied in electrodynamics is explicitly based on the flat character of the Minkowski space-time $\mathcal{E}$ and the existence of the Cartesian coordinates $\left(t, x^{a}\right), a=1,2,3$. The space-time averages are defined as follows [1]-[四].

Definition 1 The average value of a tensor field $p_{\beta}^{\alpha}(x), x \in \mathcal{E}$, over a compact space region $S$ and a finite time interval $\Delta t$ at a supporting point $\left(t, x^{a}\right) \in \Delta t \times S$ is

$$
\left\langle p_{\beta}^{\alpha}\left(t, x^{a}\right)\right\rangle_{\mathcal{E}}=\frac{1}{\Delta t V_{S}} \int_{\Delta t} \int_{S} p_{\beta}^{\alpha}\left(t+t^{\prime}, x^{a}+x^{a \prime}\right) d t^{\prime} d^{3} x^{\prime}
$$

Here $V_{S}$ is the 3-volume of the region $S$, which is usually taken as a 3-sphere of radius $R$ around the point $x^{a}$ at the instant of time $t$,

$$
V_{S}=\int_{S} d^{3} x^{\prime}
$$

Formula (1) defines the average value $\left\langle p_{\beta}^{\alpha}\left(t, x^{a}\right)\right\rangle_{\mathcal{E}}$ at a point $\left(t, x^{a}\right)$ on the manifold $\mathcal{E}$. In order to obtain averaged tensor fields and define its derivatives, it is necessary to make additional assumptions concerning the averaging regions $S$ and the intervals $\Delta t$. This assumptions are usually made only tacitly (see, however, a discussion in [1]), or they are 
$\left\langle p_{\beta}^{\alpha}\left(t, x^{a}\right)\right\rangle_{\mathcal{E}},(i i)$ all the regions $S$ and time intervals $\Delta t$ are typical in some defined sense they are usually required to be of the same shape and volume, $V_{S}=$ const and $\Delta t=$ const, and related to each other by shifting along the Cartesian coordinate lines. These properties are very easily arranged by Lie-dragging of a region $S$ and an interval $\Delta t$, chosen around a point $\left(t, x^{a}\right) \in \mathcal{E}$, along the congruences of the Cartesian coordinate lines $\mathbb{t}$ to get a "covering" of the manifold (or its connected part) with a region of the same shape and volume and a time interval of the same length around each point of $\mathcal{E}$. Consequences of the properties $(i)$ and (ii) are, first of all, the uniqueness of the definition of the average field $\left\langle p_{\beta}^{\alpha}\left(t, x^{a}\right)\right\rangle_{\mathcal{E}}$, and, secondly, the commutation formulae between the averaging and the partial derivatives

$$
\frac{\partial}{\partial t}\left\langle p_{\beta}^{\alpha}\left(t, x^{a}\right)\right\rangle_{\mathcal{E}}=\left\langle\frac{\partial}{\partial t} p_{\beta}^{\alpha}\left(t, x^{a}\right)\right\rangle_{\mathcal{E}}, \quad \frac{\partial}{\partial x^{a}}\left\langle p_{\beta}^{\alpha}\left(t, x^{a}\right)\right\rangle_{\mathcal{E}}=\left\langle\frac{\partial}{\partial x^{a}} p_{\beta}^{\alpha}\left(t, x^{a}\right)\right\rangle_{\mathcal{E}}
$$

The essence of this approach lies in the exploitation of the calculational advantages of the Cartesian coordinates on a flat manifold. The Cartesian coordinates play a central role both in defining the averages (11) and in obtaining properties, such as (2), which will allow the averaging out of the partial (ordinary) differential equations under interest. It should be stressed that by requiring $(i)$ and $(i i)$ the averages $\left\langle p_{\beta}^{\alpha}\left(t, x^{a}\right)\right\rangle_{\mathcal{E}}$ become local functions of $\left(t, x^{a}\right)$, i.e.

$$
\left(\frac{\partial}{\partial x^{a}} \frac{\partial}{\partial t}-\frac{\partial}{\partial t} \frac{\partial}{\partial x^{a}}\right)\left\langle p_{\beta}^{\alpha}\left(t, x^{a}\right)\right\rangle_{\mathcal{E}}=0
$$

and, therefore, they are provided with proper analytical properties. Furthermore, the functional dependence of the average (1) on the averaging region and interval, $S$ and $\Delta t$, becomes simply a parametric dependence on the value of the volume $V_{S}$ and the length $\Delta t$. One can, therefore, apply the standard differential and integral calculus to deal with the averages and averaged equations. The set of averaged components $\left\langle p_{\beta}^{\alpha}\left(t, x^{a}\right)\right\rangle_{\mathcal{E}}$ given by (1) in Cartesian coordinates are the components of a Lorentz tensor within the class of coordinate transformations $\widetilde{x}^{\alpha}=\Lambda_{\beta}^{\alpha} x^{\beta}+a^{\beta}$ with a constant shift $a^{\beta}$ and a constant Lorentz transformation matrix $\Lambda_{\beta}^{\alpha}$. Now, if it is necessary to consider an average field in another coordinate system, the components of $\left\langle p_{\beta}^{\alpha}\left(t, x^{a}\right)\right\rangle_{\mathcal{E}}$ in that new system are found by applying the standard tensorial transformation law.

There is still another property of the averages $\left\langle p_{\beta}^{\alpha}\left(t, x^{a}\right)\right\rangle_{\mathcal{E}}$ widely used in macroscopic electrodynamics (but again, often only tacitly assumed); the idempotency of the averages

$$
\left\langle\left\langle p_{\beta}^{\alpha}\left(t, x^{a}\right)\right\rangle_{\mathcal{E}}\right\rangle_{\mathcal{E}}=\left\langle p_{\beta}^{\alpha}\left(t, x^{a}\right)\right\rangle_{\mathcal{E}}
$$

In order to prove this property we must calculate the average value $\left\langle\left\langle p_{\beta}^{\alpha}\left(t, x^{a}\right)\right\rangle_{\mathcal{E}}\right\rangle_{\mathcal{E}}$ of the average $\left\langle p_{\beta}^{\alpha}\left(t, x^{a}\right)\right\rangle_{\mathcal{E}}$

$$
\left\langle\left\langle p_{\beta}^{\alpha}\left(t, x^{a}\right)\right\rangle_{\mathcal{E}}\right\rangle_{\mathcal{E}}=\frac{1}{\Delta t V_{S}} \int_{\Delta t} \int_{S}\left(\frac{1}{\Delta t^{\prime} V_{S^{\prime}}} \int_{\Delta t^{\prime}} \int_{S^{\prime}} p_{\beta}^{\alpha}\left(t+t^{\prime}+t^{\prime \prime}, x^{a}+x^{\prime a}+x^{\prime \prime a}\right) d t^{\prime \prime} d^{3} x^{\prime \prime}\right) d t^{\prime} d^{3} x^{\prime} .
$$

Now, the expression (5) leads to (4) under either of two additional assumptions: (iii') the averaging region $\Delta t^{\prime} \times S^{\prime}$ is the same region $\Delta t \times S$ with the supporting point at $\left(t^{\prime}, x^{\prime a}\right) \in \Delta t \times S$

${ }^{2}$ A definition of Lie-dragging, or dragging, of a region of a manifold along a vector field (congruence), or in other words, mapping of a region into another along a vector field (congruence) can be found in any 
and the average value $\left\langle p_{\beta}^{\alpha}\left(t, x^{a}\right)\right\rangle_{\mathcal{E}}$ does not depend on the choice of a supporting point within a chosen region; $\left(i i^{\prime \prime}\right)$ the averaging region $\Delta t^{\prime} \times S^{\prime}$ is a different neighbouring region and the average value $\left\langle p_{\beta}^{\alpha}\left(t, x^{a}\right)\right\rangle_{\mathcal{E}}$ remains the same if evaluated over any neighbouring regions containing a neighbourhood of $x$. Although they seem different the above assumptions are nevertheless essentially equivalent. Indeed, ( $\left.i i^{\prime}\right)$ puts emphasis on the independence of the average value with respect to the choice of a supporting point from a set of all possible points in a fixed averaging region, while $\left(i i i^{\prime \prime}\right)$ puts emphasis on the independence of the average value with respect to the choice of an averaging region from a set of all possible regions defined by a neighbourhood of a fixed supporting point. Remembering (ii) that the averaging regions are typical, change of the supporting point can be considered as change of the averaging region, and vice versa. Both assumptions thereby encode the same fundamental property of the averages which is inherently related to the philosophy of averaging itself an averaging region is considered as a point from the macroscopic point of view, and the change of either a supporting point or an averaging region does not affect the corresponding average value. The reason for this is that the differences caused by the above variations are negligibly small from a macroscopic point of view 3 .

Thus, the well-known procedure of space-time averaging in classical electrodynamics presupposes the above specific conditions $(i),($ ii $)$ and (iii) to ensure reasonable analytical and tensorial properties of the averages (1). Clearly, the whole procedure relies crucially on the existence and properties of the exceptional coordinate system in a flat space-time, namely, Cartesian coordinates. These conditions, which seem more or less trivial at first sight, require further analysis to make clear their geometrical meaning and invariant content. This will allow a reasonable formulation of a space-time averaging procedure on general (not necessarily flat) manifolds.

\section{Definition of space-time averages in macroscopic grav- ity}

Let us remind the definition of the space-time averages adopted in macroscopic gravity [14],[16]. This procedure is a generalization of the space-time averaging procedure adopted in electrodynamics (see Section 2) and it is also based on the concept of Lie-dragging of averaging regions, which makes it valid for any differentiable manifold.

Definition 2 Chosen a compact region $\Sigma \subset \mathcal{M}$ in an $n$-dimensional differentiable metric manifold $\left(\mathcal{M}, g_{\alpha \beta}\right)$ with a volume $n$-form and a supporting point $x \in \Sigma$ to which the average value will be prescribed, the average value of an object (tensor, geometric object, etc.)

\footnotetext{
${ }^{3}$ It should be noted here that in classical hydrodynamics, as distinct from macroscopic electrodynamics, discussion on the definition and properties of the averages continues for more than one hundred years. A definition of an average (either over space, time, ensemble, or a combination of such) in hydrodynamics and its properties are vital elements of the theory itself for it is clearly understood that the form of the equations depends on the definition and properties of the average. The definition (1) under conditions $(i)$ and $(i i)$ with the properties (2), (3) and (4), which are part of the Reynolds conditions in hydrodynamics, is known to result in the fundamental equations of hydrodynamics describing the dynamics of turbulence. If one of the Reynolds conditions is absent one must get different equations. For a discussion on averages and their properties in hydrodynamics, see, for example, 24] and references therein.
} 
$p_{\beta}^{\alpha}(x), x \in \mathcal{M}$, over a region $\Sigma$ at the supporting point $x \in \Sigma$ is defined as

$$
\bar{p}_{\beta}^{\alpha}(x)=\frac{1}{V_{\Sigma}} \int_{\Sigma} \mathbf{p}_{\beta}^{\alpha}\left(x, x^{\prime}\right) \sqrt{-g^{\prime}} d^{n} x^{\prime} \equiv\left\langle\mathbf{p}_{\beta}^{\alpha}\right\rangle,
$$

where $V_{\Sigma}$ is the volume of the region $\Sigma$,

$$
V_{\Sigma}=\int_{\Sigma} \sqrt{-g} d^{n} x
$$

Here the integration is carried out over all points $x^{\prime} \in \Sigma, g^{\prime}=\operatorname{det}\left(g_{\alpha \beta}\left(x^{\prime}\right)\right)$ (since the primary interest is in space-time manifolds, it is assumed that this determinant is negative, otherwise the negative sign in $\sqrt{-g}$ must be dropped) and the bold face object $\mathbf{p}_{\beta}^{\alpha}\left(x, x^{\prime}\right)$ in the integrand of (6) is a bilocal extension of the object $p_{\beta}^{\alpha}(x)$,

$$
\mathbf{p}_{\beta}^{\alpha}\left(x, x^{\prime}\right)=\mathcal{A}_{\mu^{\prime}}^{\alpha}\left(x, x^{\prime}\right) p_{\nu^{\prime}}^{\mu^{\prime}}\left(x^{\prime}\right) \mathcal{A}_{\beta}^{\nu^{\prime}}\left(x^{\prime}, x\right)
$$

by means of bilocal averaging operators $\mathcal{A}_{\beta^{\prime}}^{\alpha}\left(x, x^{\prime}\right)$ and $\mathcal{A}_{\beta}^{\alpha^{\prime}}\left(x^{\prime}, x\right)$. The averaging scheme is covariant and linear, $\left\langle a \mathbf{p}_{\beta}^{\alpha}+b \mathbf{q}_{\beta}^{\alpha}\right\rangle=a\left\langle\mathbf{p}_{\beta}^{\alpha}\right\rangle+b\left\langle\mathbf{q}_{\beta}^{\alpha}\right\rangle, a, b \in \mathrm{R}$, by construction and the averaged object $\bar{p}_{\beta}^{\alpha}$ keeps the same tensorial character as $p_{\beta}^{\alpha}$.

Let us suppose that the bilocal functions $\mathcal{A}_{\beta^{\prime}}^{\alpha}\left(x, x^{\prime}\right)$ and $\mathcal{A}_{\beta}^{\alpha^{\prime}}\left(x^{\prime}, x\right)$ are defined locally on an open subset $\mathcal{U} \subset \mathcal{M}, x, x^{\prime} \in \mathcal{U}$. In the following sections their algebraic and differential properties are formulated and analyzed to show that averaging operators with such properties do exist and also to find out the corresponding properties of the averages (6).

\section{Algebra of the averaging operator}

The following algebraic properties, which are a formalization of the properties of the spacetime averages in macroscopic electrodynamics using the language of bilocal operators, are required to hold [9], [14, [16].

Property 1 The coincidence limit of $\mathcal{A}_{\beta^{\prime}}^{\alpha}$ is

$$
\lim _{x^{\prime} \rightarrow x} \mathcal{A}_{\beta^{\prime}}^{\alpha}\left(x, x^{\prime}\right)=\delta_{\beta}^{\alpha} .
$$

Property 2 The operator $\mathcal{A}_{\beta^{\prime}}^{\alpha}$ is idempotent

$$
\mathcal{A}_{\beta^{\prime}}^{\alpha}\left(x, x^{\prime}\right) \mathcal{A}_{\gamma^{\prime \prime}}^{\beta^{\prime}}\left(x^{\prime}, x^{\prime \prime}\right)=\mathcal{A}_{\gamma^{\prime \prime}}^{\alpha}\left(x, x^{\prime \prime}\right) .
$$

These two properties imply that $\mathcal{A}_{\beta}^{\alpha^{\prime}}\left(x^{\prime}, x\right)$ is the inverse operator of $\mathcal{A}_{\beta^{\prime}}^{\alpha}\left(x, x^{\prime}\right), \mathcal{A}_{\beta^{\prime}}^{\alpha} \mathcal{A}_{\gamma}^{\beta^{\prime}}=$ $\delta_{\gamma}^{\alpha}$ and $\mathcal{A}_{\beta^{\prime}}^{\alpha} \mathcal{A}_{\alpha}^{\gamma^{\prime}}=\delta_{\beta^{\prime}}^{\gamma^{\prime}}$, and that the average tensor $\bar{p}_{\beta}^{\alpha}(x)$ takes the same value as the original tensor $p_{\beta}^{\alpha}(x), \bar{p}_{\beta}^{\alpha}(x)=p_{\beta}^{\alpha}(x)$, when the integrating region $\Sigma$ is chosen infinitesimally small, or tends to zero. This implies an additional algebraic property of the averages (6), namely, that the averaging procedure commutes with the operation of index contraction.

The idempotency (10) of the averaging operator $\mathcal{A}_{\beta^{\prime}}^{\alpha}$ is designed to provide the idempotency of the averages $(\sqrt{6})$ in macroscopic gravity 
Indeed, let us consider for simplicity a vector $v^{\alpha}(x)$ and calculate the twice averaged value $\bar{v}^{\alpha}(x)$ around the same point $x \in \mathcal{U}$ by using the definition (6)

$$
\overline{\bar{v}}^{\alpha}(x)=\frac{1}{V_{\Sigma_{x}}} \int_{\Sigma_{x}}\left(\frac{1}{V_{\Sigma_{x^{\prime}}}} \int_{\Sigma_{x^{\prime}}} \mathcal{A}_{\beta^{\prime}}^{\alpha}\left(x, x^{\prime}\right) \mathcal{A}_{\gamma^{\prime \prime}}^{\beta^{\prime}}\left(x^{\prime}, x^{\prime \prime}\right) v^{\gamma^{\prime \prime}}\left(x^{\prime \prime}\right) \sqrt{-g^{\prime \prime}} d^{n} x^{\prime \prime}\right) \sqrt{-g^{\prime}} d^{n} x^{\prime}
$$

where $\Sigma_{x^{\prime}}$ is an averaging region around the point $x^{\prime} \in \mathcal{U}$. By applying the idempotency condition (10) the expression (12) takes the form

$$
\overline{\bar{v}}^{\alpha}(x)=\frac{1}{V_{\Sigma_{x}}} \int_{\Sigma_{x}}\left(\frac{1}{V_{\Sigma_{x^{\prime}}}} \int_{\Sigma_{x^{\prime}}} \mathcal{A}_{\gamma^{\prime \prime}}^{\alpha}\left(x, x^{\prime \prime}\right) v^{\gamma^{\prime \prime}}\left(x^{\prime \prime}\right) \sqrt{-g^{\prime \prime}} d^{n} x^{\prime \prime}\right) \sqrt{-g^{\prime}} d^{n} x^{\prime} .
$$

Now, if the term in parentheses were independent of $x^{\prime}$, we could take it outside the integral with respect to the variable $x^{\prime}$ to get the resulting property (11). This term in (13), however, depends explicitly on $x^{\prime}$ in the integration region $\Sigma_{x^{\prime}}$. Similarly as in macroscopic electrodynamics, the idempotency (11) of the averages (6) follows under either of two additional assumptions [25]: $\left(i i i^{\prime}\right)$ the averaging region $\Sigma_{x^{\prime}}$ is the same region $\Sigma_{x}$ with the supporting point at $x^{\prime} \in \Sigma_{x}$ and the average value $\bar{p}_{\beta}^{\alpha}(x)$ does not depend on the choice of a supporting point within a chosen region; $\left(i i^{\prime \prime}\right)$ the averaging region $\Sigma_{x^{\prime}}$ is a different neighbouring region and the average value $\bar{p}_{\beta}^{\alpha}(x)$ remains the same if evaluated over any neighbouring region containing a neighbourhood of $x$. As it was emphasized in Section 2 this is a fundamental property of any physically reasonable classical averaging procedure and such stability of averages comes from the basic principles of averaging.

A microscopic field to be averaged is supposed to have two essentially different variation scalest, $\lambda$ and $L$, satisfying

$$
\lambda<<L,
$$

and an averaging region must be taken of an intermediate size $d$ such as

$$
\lambda<<d<<L
$$

so that the averaging effectively smooths out all the variations of the microscopic field of the scale $\lambda$. It is implicitly assumed in every averaging scheme (for example, in the averaging schemes applied in classical physics in Minkowski space-time, such as in hydrodynamics and electrodynamics - see Section 2) that the result of the averaging is insensitive to the choice of the supporting point within a fixed averaging region and it is independent of the choice of integration (averaging) region itself provided the scale $d$ satisfies the condition (15). This means that the microscopic averaging region is considered as a single "point" for the macroscopic averaged field. Such regions have been called "physically infinitesimally small" by Lorentz [26].

The trouble is, however, to perform a mathematically rigorous proof of this fact. This problem already appears in the usual averaging procedures in flat space-times and, to the best of our knowledge, remains unsolved (see, for example, a discussion in 24] for the case of hydrodynamics). Furthermore, it is not clear whether the property (11) has to be interpreted as an exact one, or it is rather an approximate property. A satisfactory formal analysis of the idempotency property of the volume averages is still lacking, though it is extensively used This problem certainly deserves further examination [27].

${ }^{4}$ The case of two scales is discussed here for the sake of simplicity. Of course, very often there is a hierarchy of scales, in which case the arguments are applied for each couple of scales satisfying (14) to be micro- and macroscopic ones. respectively. 


\section{$5 \quad$ Structure of the averaging operator}

In the previous section we discussed two algebraic Properties 11 and 2 of the bilocal operator $\mathcal{A}_{\beta^{\prime}}^{\alpha}\left(x, x^{\prime}\right)$ and the physical motivations which make these two conditions plausible. In this section the consequences of requiring (9) and (10) in the structure of the bilocal operator are analyzed. The following main theorem holds.

Theorem 1 The bilocal operator $\mathcal{A}_{\beta}^{\alpha^{\prime}}\left(x^{\prime}, x\right)$ is idempotent if and only if it is factorized. That is to say, for a bilocal operator $\mathcal{A}_{\beta}^{\alpha^{\prime}}\left(x^{\prime}, x\right)$ Properties 1 and $\mathbf{g}$ are equivalent to

$$
\mathcal{A}_{\beta}^{\alpha^{\prime}}\left(x^{\prime}, x\right)=F_{i}^{\alpha^{\prime}}\left(x^{\prime}\right) F_{\beta}^{-1^{i}}(x)
$$

where $F_{i}^{\beta}(x)$ is a set of $n$ linear independent vector fields and $F^{-1}{ }_{\beta}^{i}(x)$ is the associated dual 1 -form basis, $i=1, \ldots, n$, and summation over $i$ is carried out.

Proof. The inverse implication is checked trivially, so let us consider the direct implication only. Indeed, the partial derivative of the idempotency relation (2), $\mathcal{A}_{\alpha^{\prime}}^{\gamma^{\prime \prime}}\left(x^{\prime \prime}, x^{\prime}\right) \mathcal{A}_{\beta}^{\alpha^{\prime}}\left(x^{\prime}, x\right)=$ $\mathcal{A}_{\beta}^{\gamma^{\prime \prime}}\left(x^{\prime \prime}, x\right)$, with respect to $x^{\sigma^{\prime}}$ results in the following expression

$$
\mathcal{A}_{\alpha^{\prime}, \sigma^{\prime}}^{\gamma^{\prime \prime}}\left(x^{\prime \prime}, x^{\prime}\right) \mathcal{A}_{\beta}^{\alpha^{\prime}}\left(x^{\prime}, x\right)+\mathcal{A}_{\alpha^{\prime}}^{\gamma^{\prime \prime}}\left(x^{\prime \prime}, x^{\prime}\right) \mathcal{A}_{\beta, \sigma^{\prime}}^{\alpha^{\prime}}\left(x^{\prime}, x\right)=0 .
$$

Contracting this expression with $\mathcal{A}_{\gamma^{\prime \prime}}^{\varepsilon^{\prime}}\left(x^{\prime}, x^{\prime \prime}\right)$ and $\mathcal{A}_{\mu^{\prime}}^{\beta}\left(x, x^{\prime}\right)$ and using both (9) and (10) one gets

$$
\mathcal{A}_{\gamma^{\prime \prime}}^{\epsilon^{\prime}}\left(x^{\prime}, x^{\prime \prime}\right) \mathcal{A}_{\mu^{\prime}, \sigma^{\prime}}^{\gamma^{\prime \prime}}\left(x^{\prime \prime}, x^{\prime}\right)+\mathcal{A}_{\beta, \sigma^{\prime}}^{\epsilon^{\prime}}\left(x^{\prime}, x\right) \mathcal{A}_{\mu^{\prime}}^{\beta}\left(x, x^{\prime}\right)=0 \text {. }
$$

Now, the first summand does not depend on $x$ and the second one does not depend on $x^{\prime \prime}$, which means that

$$
\mathcal{A}_{\beta, \sigma^{\prime}}^{\epsilon^{\prime}}\left(x^{\prime}, x\right) \mathcal{A}_{\mu^{\prime}}^{\beta}\left(x, x^{\prime}\right) \equiv B_{\mu^{\prime} \sigma^{\prime}}^{\epsilon^{\prime}}\left(x^{\prime}\right),
$$

where $B_{\mu^{\prime} \sigma^{\prime}}^{\alpha^{\prime}}$ are arbitrary functions depending only on the variable $x^{\prime}$. The relation (18) immediately implies the following identity for the functions $B_{\mu^{\prime} \sigma^{\prime}}^{\alpha^{\prime}}\left(x^{\prime}\right)$ :

$$
B_{\mu^{\prime}\left[\sigma^{\prime}, \delta^{\prime}\right]}^{\epsilon^{\prime}}+B_{\rho^{\prime}\left[\sigma^{\prime}\right.}^{\epsilon^{\prime}} B_{\underline{\left.\mu^{\prime} \delta^{\prime}\right]}}^{\rho^{\prime}}=0
$$

where the square brackets denote, as usual, antisymmetrization and the underlined indices are not affected by antisymmetrization. Considering now (18) as a set of linear partial differential equations for the unknowns $\mathcal{A}_{\beta}^{\alpha^{\prime}}\left(x^{\prime}, x\right)$ ( $x$ being a parameter)

$$
\mathcal{A}_{\beta, \sigma^{\prime}}^{\epsilon^{\prime}}\left(x^{\prime}, x\right)=B_{\mu^{\prime} \sigma^{\prime}}^{\epsilon^{\prime}}\left(x^{\prime}\right) \mathcal{A}_{\beta}^{\mu^{\prime}}\left(x^{\prime}, x\right)
$$

one observes that they are always integrable because $B_{\mu^{\prime} \sigma^{\prime}}^{\alpha^{\prime}}\left(x^{\prime}\right)$ satisfy the identity (19). The general solution of the system of equations (20) is of the form

$$
\mathcal{A}_{\beta}^{\epsilon^{\prime}}\left(x^{\prime}, x\right)=F_{i}^{\epsilon^{\prime}}\left(x^{\prime}\right) H_{\beta}^{i}(x)
$$

where $F_{i}^{\alpha^{\prime}}\left(x^{\prime}\right), i=1, \ldots, n$, are $n^{2}$ independent solutions of (20) and $H_{\beta}^{i}(x)$ are $n^{2}$ arbitrary constants of integration (thereby depending on $x$ ). It only remains to impose the coincidence limit property (9) to set $H_{\beta}^{i}(x)=F^{-1}{ }_{\beta}(x)$ and the proof of the theorem is completed.

problems related with idempotency is replaced by the necessity to prove the ergodicity hypothesis which states that ensemble and time (or space) averages are equivalent. Both ensemble and volume averagings have their own advantages and areas of applicabilitv in describing phvsical phenomena. It is imnortant to 


\section{Differential properties of bilocal operators}

In this section the differential properties of the bilocal operators are summarized. This overview is essential for understanding further results concerning the space-time averaging scheme.

In order to obtain the averaged fields of the geometric objects on $\mathcal{M}$ one needs to assign an averaging region $\Sigma_{x}$ to each point $x$ of $\mathcal{U} \subset \mathcal{M}$, where the averaging integral (6) is to be evaluated. Furthermore, to calculate directional, partial and covariant derivatives of the averaged fields a law of correspondence between neighbouring averaging regions must be defined [14, [16], 21]. A natural way to define such a correspondence is to relate averaging regions by Lie-dragging, or mapping of a region into another along a vector field (see Section 2 ), by means of another bilocal operator $\mathcal{W}_{\beta}^{\alpha^{\prime}}\left(x^{\prime}, x\right)$ which is also assumed to satisfy the coincidence limit property (9).

To derive the commutation formulae between the averaging and the derivation, one should define first the directional derivative of an average field $\bar{p}_{\beta}^{\alpha}(x)$ along a vector field $\vec{\xi}=d / d \lambda$,

$$
\frac{d}{d \lambda} \bar{p}_{\beta}^{\alpha}(x)=\lim _{\Delta \lambda \rightarrow 0} \frac{1}{\Delta \lambda}\left[\bar{p}_{\beta}^{\alpha}(x+\Delta x)-\bar{p}_{\beta}^{\alpha}(x)\right]
$$

where $\Delta x^{\alpha}=\xi^{\alpha}(x) \Delta \lambda$. Let us define now the shift field for every point $x^{\prime} \in \Sigma_{x}$ as [14], [16], [21]

$$
S^{\alpha^{\prime}}\left(x^{\prime}, x\right)=\mathcal{W}_{\beta}^{\alpha^{\prime}}\left(x^{\prime}, x\right) \xi^{\beta}(x) .
$$

Now, the averaging region $\Sigma_{x+\Delta x}$ associated with the point $x+\Delta x$ is obtained by Liedragging the averaging region $\Sigma_{x}$ a parametric length $\Delta \lambda$ (the same for all $x^{\prime} \in \Sigma_{x}$ ) along the integral lines of the field $S^{\alpha^{\prime}}$.

The coordination bivector $\mathcal{W}_{\beta}^{\alpha^{\prime}}$ allows the construction of the shift vector $S^{\alpha^{\prime}}$ for any averaging region and any vector $\xi^{\alpha}$ (the shift vector at the supporting point for that region). By choosing $n$ such linearly independent vector fields $\xi_{i}^{\alpha}$ and shifting averaging regions along them one can build a covering of the manifold with one averaging region associated to every $x \in \mathcal{U} \subset \mathcal{M}$. This procedure is a formalization of the condition $(i)$ of Section 2.

As a consequence of the definitions (6), (21) and (22) one can obtain the following formula for the commutation of partial differentiation and averaging [14], [16]:

$$
\bar{p}_{\beta, \lambda}^{\alpha}=\left\langle\mathcal{A}_{\mu^{\prime}}^{\alpha} p_{\nu^{\prime}, \epsilon^{\prime}}^{\mu^{\prime}} \mathcal{A}_{\beta}^{\nu^{\prime}} \mathcal{W}_{\lambda}^{\epsilon^{\prime}}\right\rangle+\left\langle\mathbf{p}_{\beta}^{\alpha} \mathcal{W}_{\lambda: \epsilon^{\prime}}^{\epsilon^{\prime}}\right\rangle-\bar{p}_{\beta}^{\alpha}\left\langle\mathcal{W}_{\lambda: \epsilon^{\prime}}^{\epsilon^{\prime}}\right\rangle-\left\langle\mathcal{S}_{\sigma \lambda}^{\alpha} \mathbf{p}_{\beta}^{\sigma}\right\rangle+\left\langle\mathbf{p}_{\sigma}^{\alpha} \mathcal{S}_{\beta \lambda}^{\sigma}\right\rangle .
$$

Here, $\mathcal{S}_{\sigma \lambda}^{\alpha}$ are the so-called structural functions, $\mathcal{S}_{\beta \gamma}^{\alpha}=\mathcal{A}_{\epsilon^{\prime}}^{\alpha}\left(\mathcal{A}_{\beta, \gamma}^{\epsilon^{\prime}}+\mathcal{A}_{\beta, \sigma^{\prime}}^{\epsilon^{\prime}} \mathcal{W}_{\gamma}^{\sigma^{\prime}}\right)$ and $\mathcal{W}_{\lambda: \epsilon^{\prime}}^{\epsilon^{\prime}}$ is the divergence of the coordination bivector, $\mathcal{W}_{\lambda: \epsilon^{\prime}}^{\epsilon^{\prime}}=\mathcal{W}_{\lambda, \epsilon^{\prime}}^{\epsilon^{\prime}}+(\ln \sqrt{-g})_{\epsilon^{\prime}} \mathcal{W}_{\lambda}^{\epsilon^{\prime}}$. In an affine connection space with connection coefficients $\Gamma_{\beta \gamma}^{\alpha}$ (a Riemannian space is considered in [21) the same formula (23) remains valid for covariant derivatives, where partial differentiation is replaced by the covariant one and the divergence $\mathcal{W}_{\lambda: \epsilon^{\prime}}^{\epsilon^{\prime}}$ is replaced by $\mathcal{W}_{\lambda ; \epsilon^{\prime}}^{\epsilon^{\prime}}=\mathcal{W}_{\lambda, \epsilon^{\prime}}^{\epsilon^{\prime}}+\Gamma_{\epsilon^{\prime}} \mathcal{W}_{\lambda}^{\epsilon^{\prime}}$ (with $\Gamma_{\epsilon^{\prime}}=\Gamma_{\epsilon^{\prime} \alpha^{\prime}}^{\alpha^{\prime}}$ ). The commutation formula (23) has a very transparent meaning: the first term in the right-hand side is the average value of the derivative of $p_{\beta}^{\alpha}$ weighted by $\mathcal{W}_{\beta}^{\alpha^{\prime}}$, the last two terms are due to the non-triviality of the averaging operator $\mathcal{A}_{\beta}^{\alpha^{\prime}}$, while the second and third terms describe the effect of a non-trivial averaging measure in (6) and the variation in the value of the volume for different regions, respectively. Indeed, the change in the volume $V_{\Sigma}$ of an (averaging) region $\Sigma \subset \mathcal{M}$ Lie-dragged along a vector field $\xi$ is given by (see, for example, $\sqrt{20 \mid} \cdot[23]$ ) 
which can be written in terms of partial derivatives after using (22) as [25]

$$
V_{\Sigma, \beta}=\left\langle\mathcal{W}_{\beta: \alpha^{\prime}}^{\alpha^{\prime}}\right\rangle V_{\Sigma} .
$$

The expression (23) is the most general version of formulae (2) for arbitrary averaging and coordination operators.

A fundamental problem one has to face now is whether it is possible or not to define a unique covering of the manifold $\mathcal{M}$, like in Minkowski space-time (see Section 2). In other words, given a microscopic tensor field $p_{\beta}^{\alpha}(x)$, the goal is to determine a uniquely defined averaged tensor field $\bar{p}_{\beta}^{\alpha}(x)$ with reasonable analytical properties in its dependence on the supporting point. Furthermore, we must deal with the problem that the averages (6) depend functionally on the averaging regions $\bar{p}_{\beta}^{\alpha}(x)=\bar{p}_{\beta}^{\alpha}(x)[\Sigma]$ and applying the commutation formula (23) to find averaged equations would, in general, bring volume dependent terms into them, which is undesirable. In the case of Minkowski space-time the uniqueness of the averaged field with the proper analytical behaviour (3) and the parametric dependence on the averaging region volume are ensured by the condition (ii) (see Section 2) which sets the averaging regions to be typical, of the same shape and volume, $V_{S}=$ const and $\Delta t=$ const, and shifted along the Cartesian coordinate lines. To arrange similar properties for the generalized averages (6) one must look for some specific conditions on the coordination operator $\mathcal{W}_{\beta}^{\alpha^{\prime}}$. The following remarkable theorem holds [14], [16] (its version for Riemannian manifolds has been given in [21]).

Theorem 2 In the averaging region coordination by the bivector $\mathcal{W}_{\beta}^{\alpha^{\prime}}$ on an arbitrary differentiable manifold, it is necessary and sufficient to require

$$
\mathcal{W}_{[\beta, \gamma]}^{\alpha^{\prime}}+\mathcal{W}_{\left[\beta, \delta^{\prime}\right.}^{\alpha^{\prime}} \mathcal{A}_{\gamma]}^{\delta^{\prime}}=0,
$$

for the average tensor field $\bar{p}_{\beta}^{\alpha}(x)$ to be a single valued local function of the supporting point $x$ on $\mathcal{U} \subset \mathcal{M}$,

$$
\bar{p}_{\beta,[\mu \nu]}^{\alpha}=0 .
$$

Proof. The sufficiency of (26) is easily proved by calculating the antisymmetrized second partial derivative of (23) which, after using (26), gives (27). The necessity follows from the analysis of the condition (27) after taking into account that the averaging regions and the tensor field $p_{\beta}^{\alpha}$ are arbitrary.

Geometrically, (26) means that, given an averaging region $\Sigma$, the region $\widetilde{\Sigma}$ obtained by transporting $\Sigma$ along an infinitesimal parallelogram constructed from two commuting vector fields $\xi$ and $\zeta$ according to the law (22) coincides with the original region, $\widetilde{\Sigma}=\Sigma$. This is a highly non-trivial property which allows to construct a covering of the manifold with an averaging region attached to every point in the manifold, thus generalizing the corresponding part of the condition (ii) in Section 2. In the formalism of bilocal exterior calculus the condition (26) reads that the operator $\mathcal{W}_{\beta}^{\alpha^{\prime}}$ is biholonomic, which means that the bilocal coordinate 1 -form basis $\mathcal{W}^{\alpha^{\prime}}=\mathcal{W}_{\beta}^{\alpha^{\prime}} \mathrm{d} x^{\beta}$ has vanishing biholonomicity coefficients in the bilocal Maurer-Cartan equations [16].

It should be noted here that formula (27) is analogous to formula (3) in both form and meaning.

Another condition on the coordination bivector $\mathcal{W}_{\beta}^{\alpha^{\prime}}$ is the requirement that the Liedragging of a region is a volume-preserving diffeomorphism [14, [16] (21] for Riemannian 
which means that the averaging regions do not change the value of the volume when shifted (coordinated) along a chosen vector field $\xi$ according to (22). This generalizes the corresponding part of the condition (ii) in Section 2.

Thus, the condition (26) states that the average tensor field is a single valued local function of the supporting point $x$ (27). Adding the condition (28), the average tensor field does not depend explicitly on the value of the region volume $V=V_{\Sigma}$, and $V$ itself is a free parameter of the theory. Given a microscopic tensor field $p_{\beta}^{\alpha}(x)$ on $\mathcal{M}$, the average tensor field $\bar{p}_{\beta}^{\alpha}(x)$ is therefore uniquely defined on $\mathcal{U} \subset \mathcal{M}$ and can be handled within the framework of standard differential and integral calculus. Requiring additionally that the two bivectors $\mathcal{A}_{\beta}^{\alpha^{\prime}}$ and $\mathcal{W}_{\beta}^{\alpha^{\prime}}$ coincide

$$
\mathcal{A}_{\beta}^{\alpha^{\prime}}=\mathcal{W}_{\beta}^{\alpha^{\prime}}
$$

the first term in the commutation formula (23) becomes exactly the average derivative. Using all conditions (26), (28) and (29), the commutation formula acquires a remarkable simply form [14, 16]

$$
\bar{p}_{\beta, \gamma}^{\alpha}=\left\langle\mathbf{p}_{\beta, \gamma}^{\alpha}+\mathbf{p}_{\beta, \alpha^{\prime}}^{\alpha} \mathcal{W}_{\gamma}^{\alpha^{\prime}}\right\rangle .
$$

The corresponding analogues of this expression for covariant differentiation are obtained by replacing partial derivatives by covariant ones. To obtain the expression for directional derivatives we must contract this expression with a vector $\xi^{\gamma}$ and insert the vector field $S^{\alpha^{\prime}}$ from (22) in the second term of the right-hand side of (30). Formula (30) generalizes formulae (21) and it can be easily shown [16] to become exactly (2) if $\mathcal{W}_{\beta}^{\alpha^{\prime}}=\delta_{\beta}^{\alpha}$ and the volume $n$-form $\varepsilon$ is standard, that is $(\ln \sqrt{-g})_{, \epsilon^{\prime}}=0$ and $\varepsilon=\mathrm{d} x^{1} \wedge \ldots \wedge \mathrm{d} x^{n}$ [22], [23]. In Section 8 one can find more details on this particular case, see formulae (46) and (53).

\section{Existence theorems}

The differential conditions (26) and (28) together with the algebraic conditions (91), (10) and (29) are to be considered as a set of partial differential and algebraic equations for the unknown functions $\mathcal{W}_{\beta}^{\alpha^{\prime}}$. Provided a solution for the system is found, the existence of such operators $\mathcal{A}_{\beta}^{\alpha^{\prime}}$ and $\mathcal{W}_{\beta}^{\alpha^{\prime}}$ (and therefore of the averages with the above described properties) is proved. Theorem 11 has revealed the structure of the operator $\mathcal{W}_{\beta}^{\alpha^{\prime}}$ obeying the algebraic properties (9), (10). The following theorem gives the general solution of (26) (in [14], [16] and [21] for Riemannian manifolds, the same theorem has proved a solution of (26) with (29) sought in a factorized form (16), and now, with Theorem 1 taken into account, it gives the general solution).

Theorem 3 In an arbitrary n-dimensional differentiable manifold the general solution of the equations

$$
\mathcal{W}_{[\beta, \gamma]}^{\alpha^{\prime}}+\mathcal{W}_{\left[\beta, \delta^{\prime}\right.}^{\alpha^{\prime}} \mathcal{W}_{\gamma]}^{\delta^{\prime}}=0,
$$

for idempotent bilocals $\mathcal{W}_{\beta}^{\alpha^{\prime}}\left(x^{\prime}, x\right)$ is given by

$$
\mathcal{W}_{\beta}^{\alpha^{\prime}}\left(x^{\prime}, x\right)=f_{i}^{\alpha^{\prime}}\left(x^{\prime}\right) f_{\beta}^{-1^{i}}(x)
$$

where $f_{i}^{\alpha}(x) \partial_{\alpha}=\boldsymbol{f}_{\boldsymbol{i}}$ is any vector basis satisfying the commutation relations 
with constant structure functions (anholonomicity coefficients) $C_{i j}^{k}$,

$$
C_{i j}^{k}=\text { const. }
$$

Proof. Due to Theorem 1 the idempotent bivector $\mathcal{W}_{\beta}^{\alpha^{\prime}}\left(x^{\prime}, x\right)$ has the factorized form (16)

$$
\mathcal{W}_{\beta}^{\alpha^{\prime}}\left(x^{\prime}, x\right)=F_{i}^{\alpha^{\prime}}\left(x^{\prime}\right) F_{\beta}^{-1^{i}}(x) .
$$

Inserting this expression into (31) one finds

$$
F_{i}^{\alpha^{\prime}}\left(x^{\prime}\right) F_{[\beta, \gamma]}^{-1^{i}}(x)+F_{i, \delta^{\prime}}^{\alpha^{\prime}}\left(x^{\prime}\right) F_{[\beta}^{-1^{i}}(x) F_{j}^{\delta^{\prime}}\left(x^{\prime}\right) F_{\gamma]}^{-1 j}(x)=0 .
$$

Now, using the expression relating derivatives of a vector basis and its dual 1-form basis

$$
F_{j, \mu^{\prime}}^{\alpha^{\prime}}=-F_{l}^{\alpha^{\prime}} F_{\beta^{\prime}, \mu^{\prime}}^{-F_{j}^{l}} F_{j}^{-\beta^{\prime}}
$$

in equation (35), it becomes

$$
2 F_{l}^{\rho} F_{k}^{\sigma} F_{[\rho, \sigma]}^{-1^{i}}(x)=2 F_{l}^{\rho^{\prime}} F_{k}^{\sigma^{\prime}} F_{\left[\rho^{\prime}, \sigma^{\prime}\right]}^{-1^{i}}\left(x^{\prime}\right) \equiv C_{l k}^{i},
$$

which is exactly the expression for the anholonomicity coefficients $C_{l k}^{i}$ in terms of a 1-form basis. Equations (37) say that any vector basis $F_{i}^{\alpha}(x)=f_{i}^{\alpha}(x)$ such that its corresponding dual 1-form basis $\boldsymbol{f}^{-\mathbf{1}^{i}}=f_{\alpha}^{-1^{i}} \mathrm{~d} x^{\alpha}$ satisfies the Maurer-Cartan equation

$$
\mathrm{d} \boldsymbol{f}^{-1^{i}}=-\frac{1}{2} C_{j k}^{i} \boldsymbol{f}^{-\mathbf{1}^{j}} \wedge \boldsymbol{f}^{-\mathbf{1}^{k}}
$$

with constant anholonomicity coefficients $C_{l k}^{i}=$ const, is a solution of (31). The class of all such bases constitutes the general solution of equations (31) and therefore the general solution for the coordination bivector satisfying (26) and (29).

The next theorem proves the existence of solutions for the equation (28) within the class of bivectors satisfying (32) [25] (its version for a particular subclass of (32), see Section 8 and Proposition 1 below, has been given in [14], [16], and [21] for the case of Riemannian manifolds).

Theorem 4 In an arbitrary $n$-dimensional differentiable metric manifold $\left(\mathcal{M}, g_{\alpha \beta}\right)$ with a volume $n$-form there always exist locally volume-preserving bivectors $\mathcal{W}_{\beta}^{\alpha^{\prime}}\left(x^{\prime}, x\right)$ of the form (32) with (34) satisfying (28).

Proof. For a bivector $\mathcal{W}_{\beta}^{\alpha^{\prime}}\left(x^{\prime}, x\right)=f_{i}^{\alpha^{\prime}}\left(x^{\prime}\right) f_{\beta}^{-1^{i}}(x)$ equations (28) read

$$
f_{i: \alpha^{\prime}}^{\alpha^{\prime}} \equiv f_{i, \alpha^{\prime}}^{\alpha^{\prime}}+(\ln \sqrt{-g})_{\alpha^{\prime}} f_{i}^{\alpha^{\prime}}=0 .
$$

Writing (39) as

$$
-f_{i}^{\alpha^{\prime}} f_{\beta^{\prime}, \alpha^{\prime}}^{-1^{i}}+(\ln \sqrt{-g})_{, \beta^{\prime}}=0,
$$

and using the definition of the anholonomicity coefficients in terms of 1-form basis (see (37) above) this equation can be rewritten as: 
Now it is sufficient to show the integrability of this quasi-linear partial differential equation to prove the theorem. Taking into account (34) and the property $C_{j i}^{i} C_{k l}^{j}=0$ which holds in that case, one can easily show that (41) is always integrable on an arbitrary $n$-dimensional differentiable manifold with a volume $n$-form.

It should be stressed here that the conditions $C_{j k}^{i}=$ const due to Theorem 3 are essential for the proof of Theorem 1 and they guarantee the local existence of $n$ linear independent divergence free vectors, the result holding for both orientable and non-orientable manifolds (by using the so-called odd volume $n$-form [28], [29]) )

Another important point is that $n$ vector fields $f_{i}^{\alpha}$ satisfying (33) and (34) define a finite dimensional Lie group on the averaged manifolds which is directly related with the symmetries of such manifolds [31].

These two theorems prove the existence of solutions for the set of equations (91), (10), (26), (28), and (29), and therefore prove the existence of the bilocal operator $\mathcal{W}_{\beta}^{\alpha^{\prime}}\left(x^{\prime}, x\right)$ with the corresponding algebraic and differential properties (27) and (30) for the averages (6).

Now a particular subclass of the operators (32) with (34) will be considered to analyze some additional properties and to reveal the functional structure of the subclass.

\section{The proper systems of coordinates}

As it has been emphasized in the Introduction and Section 2, the space-time averaging procedure adopted in electrodynamics is essentially formulated in Cartesian coordinates and all its properties are shown by exploiting the exceptional character of this coordinates (see Section 2 for details). The covariant formalism developed for the averages (6) in macroscopic gravity (Sections 3-6) generalizes the averaging scheme of macroscopic electrodynamics for arbitrary $n$-dimensional differentiable manifolds and while keeping covariant properties which are analogous to those in electrodynamics.

Let us now consider the macroscopic gravity averaging scheme for a particular subclass of operators (32) with (34). This particular subclass admits a special coordinate system in which the averages and their properties have especially simple form and meaning. Such a coordinate system is an analogue for macroscopic gravity of the Cartesian coordinates in Minkowski space-time.

Let us hereby restrict the class of solutions of the equations (31) to the subclass satisfying

$$
\left[\boldsymbol{f}_{\boldsymbol{i}}, \boldsymbol{f}_{\boldsymbol{j}}\right]=0
$$

that is $C_{i j}^{k} \equiv 0$. In this case the vector fields $f_{i}^{\alpha}$ constitute a coordinate system and there always exist $n$ functionally independent scalar functions $\phi^{i}(x)$ such that the vector and corresponding dual 1-form bases are of the form

$$
f_{i}^{\alpha}\left(x\left(\phi^{k}\right)\right)=\frac{\partial x^{\alpha}}{\partial \phi^{i}}, \quad f_{\alpha}^{-1^{i}}\left(\phi\left(x^{\mu}\right)\right)=\frac{\partial \phi^{i}}{\partial x^{\alpha}} .
$$

\footnotetext{
${ }^{6} n$ linear independent divergence free vector fields on an $n$-dimensional differentiable manifold with a volume $n$-form with structure functions (33) can be shown to exist locally iff $C_{l k, i}^{i}=0$. This condition is fulfilled also globally on parallelizable manifolds (both orientable and non-orientable) [30]. This shows that Theorem 1 is valid globally for such manifolds (a manifold is called parallelizable if its tangent bundle is
} 
Thus, the bilocal operator $\mathcal{W}_{\beta}^{\alpha^{\prime}}\left(x^{\prime}, x\right)$ becomes

$$
\mathcal{W}_{\beta}^{\alpha^{\prime}}\left(x^{\prime}, x\right)=\frac{\partial x^{\alpha^{\prime}}}{\partial \phi^{i}} \frac{\partial \phi^{i}}{\partial x^{\beta}} .
$$

Being functionally independent, the set of $n$ functions $\phi^{i}(x)$ can be taken as a system of local coordinates on the manifold $\mathcal{M}$ [14], [16] (21] for Riemannian manifolds).

Definition 3 A coordinate system $\left\{\phi^{i}\right\}$ defined by $n$ scalar functions $\phi^{i}=\phi^{i}(x)$ in (43) will be called a proper coordinate system.

The usefulness of this definition is motivated by the fact that in a proper coordinate system the bilocal operator $\mathcal{W}_{\beta}^{\alpha^{\prime}}\left(x^{\prime}, x\right)$ takes the simplest possible form

$$
\mathcal{W}_{j}^{i}\left(\phi^{\prime}, \phi\right) \equiv \mathcal{W}_{\beta}^{\alpha^{\prime}}\left(x^{\prime}, x\right)_{\mid x^{\alpha}=\phi^{i}}=\boldsymbol{\delta}_{\beta}^{\alpha^{\prime}} \equiv \boldsymbol{\delta}_{j}^{i},
$$

where the bilocal Kronecker symbol $\boldsymbol{\delta}_{\beta}^{\alpha^{\prime}}$ is defined as $\boldsymbol{\delta}_{\beta}^{\alpha^{\prime}}=\delta_{i}^{\alpha^{\prime}} \delta_{\beta}^{i}$. The definition of the average (6) acquires a remarkable simple form (closely resembling the space-time averages of macroscopic electrodynamics (1)) when written using a proper coordinate system

$$
\bar{p}_{j}^{i}(\phi)=\frac{1}{V_{\Sigma_{\phi}}} \int_{\Sigma_{\phi}} p_{j}^{i}\left(\phi^{\prime}\right) \sqrt{-g\left(\phi^{\prime}\right)} d^{n} \phi^{\prime} .
$$

Theorem 14 has proved the existence of volume-preserving bilocal operators (32) and (34), and therefore the existence of solutions of (28) for operators $\mathcal{W}_{\beta}^{\alpha^{\prime}}$ of the form (44) from this theorem. It is useful, however, to prove it here independently due to the above mentioned importance of the proper system of coordinates for the macroscopic gravity averaging scheme. The following statement holds in this case [14], [16] (21] for Riemannian manifolds).

Proposition 1 In an arbitrary n-dimensional differentiable metric manifold $\left(\mathcal{M}, g_{\alpha \beta}\right)$ with a volume $n$-form, there always exist locally a set of $n$ scalar functionally independent functions $\phi^{i}(x)$ such that the corresponding coordination bivector (44) satisfies condition (28).

Proof. Let us write down equations (28) for the bivector $\mathcal{W}_{\beta}^{\alpha^{\prime}}$ (44)

$$
\frac{\partial^{2} x^{\alpha^{\prime}}}{\partial \phi^{i} \partial \phi^{j}} \frac{\partial \phi^{j}}{\partial x^{\alpha^{\prime}}}+\left(\ln \sqrt{-g\left(x^{\prime}\right)}\right)_{, \mu^{\prime}} \frac{\partial x^{\mu^{\prime}}}{\partial \phi^{i}}=0
$$

in terms of the unknowns $\phi^{i}(x)$. These equations are equivalent to the system of quasi-linear partial differential equations (compare with (41)) for $f_{\alpha}^{-1^{i}}$ (43)

$$
-f_{i}^{\alpha^{\prime}}\left(x^{\prime}\left(\phi^{k}\right)\right) f_{\alpha^{\prime}, j}^{-1^{i}}\left(\phi^{k}\right)+\left(\ln \sqrt{-g\left(x^{\prime}\left(\phi^{k}\right)\right)}\right)_{, j}=0
$$

which are always locally integrable on an arbitrary $n$-dimensional differentiable manifold with a volume $n$-form. Then, in accordance with (43) $\phi^{i}(x)$ are solutions of the equations $\phi_{, \alpha}^{i}=f^{-1^{i}}$, which are also always locally integrable because the integrability conditions 
Corollary 1 Any proper coordinate coordinate system such that the corresponding bivector (44) satisfies condition (28) is a volume-preserving system of coordinates,

$$
\left(\ln \sqrt{-g\left(\phi^{k}\right)}\right)_{, j}=0, \quad \text { or }, \quad g\left(\phi^{k}\right)=\text { const } .
$$

It should be noted here that all arguments concerning non-orientable manifolds and global existence given after Theorem 10 apply here as well (see Section 7).

For the case of (pseudo)-Riemannian manifolds, Corollary 1 states that in a proper coordinate system the Christoffel symbols $\Gamma_{\beta \alpha}^{\alpha}$, (which are $\Gamma_{\beta \alpha}^{\alpha}=(\ln \sqrt{-g})_{, \beta}$ due to equi-affinity of Riemannian manifolds) vanish

$$
\Gamma_{i}(\phi) \equiv \Gamma_{i j}^{j}=\left(\ln \sqrt{-g\left(\phi^{k}\right)}\right)_{, j}=0 .
$$

Another useful characterization of the volume-preserving coordinates, in addition to (49), or (50), can be obtained in terms of the expansion of the vector fields tangent to the coordinates lines. Defining a vector $\chi_{(i)}$ tangent to a coordinate line $\phi^{i}$ as

$$
\chi_{(i)}=\chi_{(i)}^{j} \frac{\partial}{\partial \phi^{j}}=\delta_{(i)}^{j} \frac{\partial}{\partial \phi^{j}},
$$

it is immediate to find that in the proper coordinate system $\left\{\phi^{i}\right\}$ the expansion $\operatorname{div} \chi_{(i)}$ of the vector field (51) is

$$
\chi_{(i): j}^{j}=\left(\ln \sqrt{-g\left(\phi^{k}\right)}\right)_{, i}=0,
$$

so that the condition of vanishing expansion for the tangent vector fields $\chi_{(i)}$ is equivalent to the definition of volume-preserving coordinates (49).

In addition to very simple and transparent forms of the coordination bivector (44) and the averages (46), the volume-preserving proper coordinate systems also allow a remarkably simple expression for the commutation between partial differentiation and averaging. Indeed, expression (30) becomes in the proper coordinate system

$$
\frac{\partial}{\partial \phi^{k}} \bar{p}_{j}^{i}(\phi)=\left\langle\frac{\partial}{\partial \phi^{\prime k}} p_{j}^{i}\left(\phi^{\prime}\right)\right\rangle,
$$

which is exactly the same commutation formula for as in the averaging scheme in Minkowski manifolds (2).

Let us now study the functional structure of the class of the volume-preserving coordinates to understand how large it is and how much freedom for coordinate transformations it contains. The following Proposition reveals the structure of the class.

Proposition 2 The class of volume-preserving coordinate transformations on an arbitrary $n$-dimensional differentiable metric manifold $\left(\mathcal{M}, g_{\alpha \beta}\right)$ with a volume $n$-form, contains $(n-1)$ arbitrary functions of $n$ arguments and one arbitrary function of $(n-1)$ arguments.

Proof. Due to Proposition 10 the volume-preserving coordinates are characterized by the condition (49). The class of coordinate transformations $y^{\alpha}=y^{\alpha}\left(x^{\mu}\right)$ which preserve this condition, 
is defined by the system of partial differential equations 32]

$$
\frac{\partial^{2} y^{\alpha}}{\partial x^{\nu} \partial x^{\mu}} \frac{\partial x^{\mu}}{\partial y^{\alpha}}=0
$$

Equations (55) can be easily written as equations for the Jacobian of the coordinate transformation $y^{\alpha}=y^{\alpha}\left(x^{\mu}\right)$,

$$
\frac{\partial}{\partial x^{\nu}}\left[\operatorname{det}\left(\frac{\partial y^{\alpha}}{\partial x^{\mu}}\right)\right]=0
$$

which have the general solution

$$
\operatorname{det}\left(\frac{\partial y^{\alpha}}{\partial x^{\mu}}\right)=C
$$

where $C$ is a non-vanishing constant to ensure invertibility of the coordinate transformation. Expanding the Jacobian (57) by its first row we get

$$
\frac{\partial y^{1}}{\partial x^{1}} \xi^{1}\left(y^{2}, \ldots, y^{n}\right)+\ldots+\frac{\partial y^{1}}{\partial x^{n}} \xi^{n}\left(y^{2}, \ldots, y^{n}\right)=C
$$

where $\xi^{\nu}\left(y^{2}, \ldots, y^{n}\right)$ are the corresponding minors of the determinant, depending therefore only on $(n-1)$ functions $y^{2}, \ldots, y^{n}$. These $(n-1)$ functions are arbitrary functionally independent functions of $x^{\mu}$, and $\xi^{\nu}$ can be taken as explicit functions of $x^{\mu}, \xi^{\nu}=\xi^{\nu}\left(x^{\mu}\right)$. Then equation (58) reads

$$
\xi^{\nu}\left(x^{\mu}\right) \frac{\partial y^{1}}{\partial x^{\nu}}=C .
$$

This is a linear partial differential equation for the unknown $y^{1}\left(x^{\mu}\right)$. Its general solution is known to be

$$
y^{1}\left(x^{\mu}\right)=y_{p}^{1}\left(x^{\mu}\right)+h\left(k^{2}\left(x^{\mu}\right), \ldots, k^{n}\left(x^{\mu}\right)\right)
$$

where $y_{p}^{1}\left(x^{\mu}\right)$ is a particular solution of (59), $k^{A}\left(x^{\mu}\right), A=2, \ldots, n$, are $n-1$ functionally independent first integrals satisfying

$$
\xi^{\nu} \frac{\partial k^{A}}{\partial x^{\nu}}=0
$$

and $h$ is an arbitrary function of $(n-1)$ arguments $k^{A}$. Thus, the general solution of the equations (55) defining the class of volume-preserving coordinates (54) contains $(n-1)$ arbitrary functions $y^{A}\left(x^{\mu}\right)$ of $n$ arguments $x^{\mu}$ and one arbitrary function $h\left(k^{A}\right)$ of $(n-1)$ variables $k^{A}\left(x^{\mu}\right)$.

The set of proper coordinate systems forms quite a big class and this functional freedom may be used to specify additional properties of the averages (6), or (46), when necessary.

Choosing different proper coordinate systems $\phi^{i}$ will give different average fields (46) of a given microscopic tensor field $p_{\beta}^{\alpha}(x)$. In general, the averages $\bar{p}_{j}^{i}(\phi)$ and $\bar{p}_{j}^{i}(\widetilde{\phi})$ calculated in the proper coordinate systems $\phi^{i}$ and $\tilde{\phi}^{i}$ are not related by a tensorial law under the transformation $\widetilde{\phi}^{i}=\widetilde{\phi}^{i}\left(\phi^{j}\right)$, nor are the operators $\mathcal{W}_{j}^{i}\left(\phi^{\prime}, \phi\right)$ and $\mathcal{W}_{j}^{i}\left(\widetilde{\phi}^{\prime}, \widetilde{\phi}\right)$ related by a tensorial transformation with each of them being $\boldsymbol{\delta}_{j}^{i}(45)$ in its own proper coordinates system. It should be noted here that averages (6), and (46), are obviously tensorial with 
(32), or (44), of the bilocal operator $\mathcal{W}_{\beta}^{\alpha^{\prime}}\left(x^{\prime}, x\right)$ itself, which involves a functional freedom in changing the functions $f_{i}^{\alpha}(x)$, or $\phi^{i}(x)$. This "non-tensorial" property is very natural, indeed, for it states the exceptional character of the proper coordinate systems for obtaining the simplest and most transparent form of the averages and the averaging and coordination operators. It closely resembles, on the other hand, the definition of averages in macroscopic electrodynamics as it was noted above, and the exceptional character of Cartesian coordinates used in that averaging procedure. The class of the proper coordinate systems on an arbitrary differentiable metric manifold is a natural counterpart of the Cartesian coordinate system on a Minkowski manifold. The property they share in common is that both are volume-preserving.

There is, however, a special subclass within the class of volume-preserving coordinate transformations described in Proposition 2 which keeps the bilocal operator (45) and the averages (46) covariant.

Proposition 3 The class of transformations $\phi^{i} \rightarrow \tilde{\phi}^{i}$ which keeps the bivector $\mathcal{W}_{\beta}^{\alpha^{\prime}}$ and the averages (6) covariant within the class of proper system of coordinates is

$$
\tilde{\phi}^{i}=\Lambda_{j}^{i} \phi^{j}+a^{i}
$$

where $\Lambda_{j}^{i}$ and $a^{i}$ are constant.

Proof. The proof is straightforward. Consider two proper coordinate systems $\phi^{i}$ and $\widetilde{\phi}^{i}$, and require the bivectors $\mathcal{W}_{j}^{i}\left(\phi^{\prime}, \phi\right)$ and $\mathcal{W}_{j}^{i}\left(\widetilde{\phi}^{\prime}, \widetilde{\phi}\right)$, each of the form (45) in its own proper coordinates, to be equal

$$
\frac{\partial x^{\alpha^{\prime}}}{\partial \phi^{i}} \frac{\partial \phi^{i}}{\partial x^{\beta}}=\frac{\partial x^{\alpha^{\prime}}}{\partial \tilde{\phi}^{i}} \frac{\partial \tilde{\phi}^{i}}{\partial x^{\beta}} .
$$

Since the coordinate system $x^{\alpha}$ in equation (63) is proper by assumption it must be either $\phi^{i}$ or $\widetilde{\phi}^{i}$. Choosing $\phi^{i}$ one immediately obtains

$$
\boldsymbol{\delta}_{j}^{i}=\frac{\partial \phi^{i}}{\partial \tilde{\phi}^{l}}{ }_{\mid \tilde{\phi}\left(x^{\prime}\right)} \frac{\partial \tilde{\phi}^{l}}{\partial \phi^{j}}{ }_{\mid \phi(x)} .
$$

This last equation has as general solution (62)). It is easy to show that the class of transformations between proper coordinates keeps the averages (46) also covariant.

Due to Proposition o 2 , if the manifold $\left(\mathcal{M}, g_{\alpha \beta}\right)$ is chosen to be a (pseudo)-Riemannian spacetime, the averages (46) defined in proper coordinates are Lorentz tensors exactly like the averages in Minkowski space-time (see Section 2).

\section{Conclusion}

Thus, the covariant averaging procedure for objects (tensors, geometric objects, etc.) in the framework of macroscopic gravity [9], [14], [16] is a natural generalization of the space-time averaging procedure of macroscopic electrodynamics. It gives a covariant formulation of the conditions on the averages, which provide them with natural algebraic and analytical properties. A wide class of averaging and coordination bilocal operators satisfying all the proper- 
spaces. The class of proper coordinate systems, analogous to Cartesian coordinate system of Minkowski space-time and generalizing them, gives the simplest and most transparent form of the averages. The averaging procedure as it is formulated allows a large functional freedom which is incorporated in an elegant way and can be used to arrange additional specific conditions for the averages.

\section{Acknowledgments}

MM wishes to thank the Ministerio de Educación y Cultura (Spain) for financial support under grant EX95 40985713. RMZ was supported by a Royal Society fellowship (London, UK) and a Śniadeccy Fellowship (Cracow, Poland) and would like to thank the School of Mathematical Sciences and the N. Copernicus Astronomical Center where the work has been done in parts for hospitality. RMZ appreciates many interesting discussions with Boris Dubrovin. 


\section{References}

[1] V. Novacu, Introducere în Electrodinamică (Bucharest, Editura Academiei, 1955) (in Romanian).

[2] W.K.H. Panovsky and M. Phillips, Classical Electricity and Magnetism (AddisonWesley, Reading, 1962).

[3] L. Rosenfeld, Theory of Electrons (Addison-Wesley, Reading, 1962).

[4] R.S. Ingarden and A. Jamiołkowski, Classical Electrodynamics (PWN, Warszawa and Elsevier, Amsterdam, 1985).

[5] S.R. de Groot, The Maxwell Equations (North-Holland, Amsterdam, 1969).

[6] G. Russakoff, Amer. J. Phys. 38 (1970) 1188.

[7] F.N.H. Robinson, Macroscopic Electrodynamics (Pergamon Press, Oxford, 1973).

[8] J.D. Jackson, Classical Electrodynamics (John Wiley \& Sons, New York, 1975).

[9] R.M. Zalaletdinov, Averaging problem in general relativity, macroscopic gravity and using Einstein's equations in cosmology, Preprint, School of Mathematical Sciences, Queen Mary \& Westfield College, No. QMW-AU-96018 (London, March 1996), submitted to Phys. Rev. D.

[10] N. Bohr and L. Rosenfeld, Mat.-fys. Medd. Dan. Vid. Selsk. 12, no. 8 (1933) [English translation in Selected Papers of Léon Rosenfeld, eds. R.S. Cohen and J.J. Stachel (D. Reidel, Dordrecht, 1979) p. 357].

[11] B.S. DeWitt, in Gravitation: An introduction to current research, ed. L. Witten (Wiley, New York, 1962), p. 266.

[12] C.W. Misner, K.S. Thorne and J.A. Wheeler, Gravitation (Freeman, San Francisco, 1973)

[13] G.F.R. Ellis, in General Relativity and Gravitation, eds. B. Bertotti, F. de Felici and A. Pascolini (Reidel, Dordrecht, 1984), p. 215.

[14] R.M. Zalaletdinov, Gen. Rel. Grav. 24 (1992) 1015.

[15] A. Krasiński, Inhomogeneous Cosmological Models (Cambridge University Press, Cambridge, 1997), in press.

[16] R.M. Zalaletdinov, Gen. Rel. Grav. 25 (1993) 673.

[17] R.M. Zalaletdinov, in Proceedings of International Symposium on Experimental Gravitation, eds. M. Karim and A. Qadir (IOP, Bristol, 1994) p. A363.

[18] R.M. Zalaletdinov, In: Inhomogeneous Cosmological Models, eds. A. Molina and J.M.M. Senovilla (World Scientific, Singapore, 1995), p. 91. 
[19] R.M. Zalaletdinov, Macroscopic gravity, In: Proc. of 7th Marcel Grossmann Meeting (World Scientific, Singapore, 1996), to appear.

[20] L.Ya. Arifov, R.M. Zalaletdinov and A.V. Shein, Averaging out tensor fields on Riemannian manifolds according to Lorentz. II. Commutation formulae for the averaging and derivation (absolute and covariant). Preprint, Institute of Nuclear Physics, Uzbek Academy of Sciences, No. R-12-480 (Tashkent, 1990) (in Russian).

[21] L.Ya. Arifov, R.M. Zalaletdinov and A.V. Shein, Averaging out tensor fields on Riemannian manifolds according to Lorentz. III. Analysis of the averages. Preprint, Institute of Nuclear Physics, Uzbek Academy of Sciences, No. R-12-499 (Tashkent, 1990) (in Russian).

[22] S. Kobayashi and K. Nomizu, Foundations of Differential Geometry, Vol. I (Interscience, New York, 1963).

[23] B.F. Schutz, Geometrical Methods of Mathematical Physics (Cambridge University Press, Cambridge, 1980).

[24] A.S. Monin and A.M. Yaglom, Statistical Fluid Mechanics: Mechanics of Turbulence, Vol. 1 (Nauka, Moscow, 1965) (in Russian) [English translation of Vol.1 revised by the authors, ed. J.L. Lumley (MIT, Cambridge, Mass., 1971)].

[25] R.M. Zalaletdinov, unpublished.

[26] H.A. Lorentz, The Theory of Electrons (Teubner, Leipzig, 1916).

[27] M. Mars and R.M. Zalaletdinov, Fibre bundle formulation of space-time averaging schemes, in progress.

[28] G. de Rham, Variétés Différentiables, Actualités Scientifiques et Industrielles 1222 (Hermann, Paris, 1960).

[29] J. Moser, Trans. Amer. Math. Soc. 120 (1965) 286.

[30] M. Gromov, Partial Differential Relations (Springer-Verlag, Berlin, 1986).

[31] R.M Zalaletdinov, Space-time averaging of classical physical fields, in progress.

[32] R.M Zalaletdinov, R.K. Tavakol and G.F.R. Ellis, Gen. Rel. Grav. 28 (1996) 1251. 